\title{
Predicting Infiltrative Hepatocellular Carcinoma Patient Outcome Post-TACE: MR Bias Field Correction Effect on 3D-quantitative Image Analysis
}

\author{
Cuihong Liu ${ }^{1,2}$, Susanne Smolka ${ }^{2,3}$, Xenophon Papademetris ${ }^{4}$, Duc Do Minh ${ }^{2,3}$, Geliang Gan ${ }^{5}$, \\ Yanhong Deng ${ }^{5}$, MingDe Lin $^{2}$, Julius Chapiro ${ }^{2 *}$, Ximing Wang*6,7, Christos Georgiades ${ }^{8}$ \\ and Kelvin Hong ${ }^{8}$
}

\begin{abstract}
${ }^{1}$ Department of Ultrasound Diagnosis and Treatment, Shandong Provincial Hospital, Cheeloo College of Medicine, Shandong University, Jinan, Shandong, China; ${ }^{2}$ Department of Radiology and Biomedical Imaging, Yale University School of Medicine, New Haven, CT, USA; ${ }^{3}$ Department of Diagnostic and Interventional Radiology, Charité University Hospital, Berlin, Germany; ${ }^{4}$ Department of Biomedical Engineering, Yale University, New Haven, CT, USA; ${ }^{5}$ School of Public Health, Yale University, New Haven, CT, USA; ${ }^{6}$ Department of Radiology, Shandong Provincial Hospital, Cheeloo College of Medicine, Shandong University, Jinan, Shandong, China; ${ }^{7}$ Department of Radiology, Shandong Provincial Hospital Affiliated to Shandong First Medical University, Jinan, Shandong, China; ${ }^{8}$ Russell H. Morgan Department of Radiology and Radiological Science, Division of Vascular and Interventional Radiology, The Johns Hopkins Hospital, Baltimore, MD, USA
\end{abstract}

\begin{abstract}
Background and Aims: To investigate the impact of MR bias field correction on response determination and survival prediction using volumetric tumor enhancement analysis in patients with infiltrative hepatocellular carcinoma, after transcatheter arterial chemoembolization (TACE). Methods: This study included 101 patients treated with conventional or drug-eluting beads TACE between the years of 2001 and 2013. Semi-automated 3D quantification software was used to segment and calculate the enhancing tumor volume (ETV) of the liver with and without bias-field correction on multiphasic contrast-enhanced MRI before and 1-month after initial TACE. ETV (expressed as $\mathrm{cm}^{3}$ ) at baseline imaging and the relative change in ETV (as \% change, ETV\%) before and after TACE were used to predict response and survival, respectively. Statistical survival analyses included Kaplan-Meier curve generation and Cox proportional hazards modeling. Q statistics were calculated and used to identify the best cut-off value for ETV to separate responders and non-responders $\left(E T V \mathrm{~cm}^{3}\right)$. The difference in survival was evaluated between responders and non-responders using Kaplan-Meier and Cox models. Results: MR bias field correction correlated with improved response calculation from baseline MR as well as survival after TACE; using a $415 \mathrm{~cm}^{3}$ cut-off for ETV at baseline (hazard ratio: $2.00,95 \%$ confidence interval: 1.23-3.26, $p=0.01)$ resulted in significantly improved response prediction (median survival in patients with baseline ETV $<415 \mathrm{~cm}^{3}$ :
\end{abstract}

Keywords: 3D volume measurement; Infiltrative HCC; TACE; Segmentation; Bias field correction.

Abbreviations: ETV, enhancing tumor volume; HCC, hepatocellular carcinoma; $N R$, non-responder; OS, overall survival; $R$, responder; TACE, transcatheter arterial chemoembolization.

Received: 13 June 2020; Revised: 13 July 2020; Accepted: 20 July 2020

*Correspondence to: Julius Chapiro, Department of Radiology and Biomedica Imaging, Yale University School of Medicine, 333 Cedar Street, New Haven, CT 06520, USA. Tel: +1-203-343-7457, E-mail: j.chapiro@googlemail.com; Ximing Wang, Department of Radiology, Shandong Provincial Hospital Affiliated to Shandong First Medical University, 324 Jingwu Road, Jinan, Shandong 250021, China. Tel: +86-151-6888-7762, E-mail: wxming369@163.com
19.66 months vs. $\geq 415 \mathrm{~cm}^{3}: 9.21$ months, $p<0.001$, logrank test). $A \geq 41 \%$ relative decrease in ETV (hazard ratio: $0.58,95 \%$ confidence interval: $0.37-0.93, p=0.02$ ) was significant in predicting survival (ETV $\geq 41 \%$ : 19.20 months vs. ETV <41\%: 8.71 months, $p=0.008$, log-rank test). Without MR bias field correction, response from baseline ETV could be predicted but survival after TACE could not. Conclusions: MR bias field correction improves both response assessment and accuracy of survival prediction using whole liver tumor enhancement analysis from baseline MR after initial TACE in patients with infiltrative hepatocellular carcinoma.

Citation of this article: Liu C, Smolka S, Papademetris X, Minh DD, Gan G, Deng Y, et al. Predicting infiltrative hepatocellular carcinoma patient outcome post-TACE: MR bias field correction effect on 3D-quantitative image analysis. J Clin Transl Hepatol 2020;8(3):292-298. doi: 10.14218/ JCTH.2020.00054.

\section{Introduction}

Hepatocellular carcinoma (HCC) is the fifth most common cancer and the second leading cause of cancer-related death worldwide. ${ }^{1}$ Patients in the United States and Europe with this type of cancer have the third lowest 5-year survival rate of all other cancer types. ${ }^{2}$ The morphology of HCC can be classified into three groups: multi-nodular, massive, and infiltrative. ${ }^{3}$ Infiltrative HCC represents $7-20 \%$ of all HCC cases $^{4}$ and it is characterized by multifocal growth pattern, inhomogeneous expansion into liver parenchyma, as well as portal vein thrombosis in most cases, resulting in an advanced stage classification at initial diagnosis and a higher mortality. 5,6 Thus, most patients with infiltrative $\mathrm{HCC}$ are ineligible for curative therapies, like ablation, resection, or transplantation. ${ }^{7}$ Catheter-based intra-arterial therapies, such as transarterial chemoembolization (TACE), are commonly used as palliative treatment in patients with infiltrative HCC.

In our previous work, ${ }^{8}$ we demonstrated that, for patients with unresectable HCC treated with TACE, 3D quantitative 
enhancement-based assessment (quantitative European Association for the Study of the Liver, qEASL) could define treatment response and non-response better than traditional uni- and bi-dimensional methods, such as RECIST, mRECIST, World Health Organization and EASL. Moreover, a significant reduction of enhancing tumor volume (ETV) was found to correspond with a significant improvement in overall survival (OS) according to qEASL. ${ }^{9}$ Hence, the early and reliable identification of responder (R) and non-responder (NR) by qEASL after TACE enables treatment decisions to be made sooner. ${ }^{10,11}$

MR imaging is the reference standard and routinely used in clinical practice for the diagnosis of HCC and the assessment of TACE therapy. However, due to imperfections in the radio frequency coil, eddy currents or patient anatomy, bias field effects, presenting as a slowly varying intensity variation across the image, are common during MR imaging. The appearance of bias field is often seen as varying bands of artificial image hyper- and hypo-enhancement across the MR image. ${ }^{12}$ Put another way, an image with uniform intensity would appear to have artificial regions of increased and/or decreased image enhancement. Such image artifact could limit the accuracy of image interpretation, segmentation, and quantification. ${ }^{13,14} \mathrm{~A}$ characteristic presentation of infiltrative HCC is its diffuse appearance/ill-defined morphology that typically encompasses a large portion of the liver with absence of discrete tumor-liver parenchyma boundaries. As such, the MR bias field artifact can interfere with and exacerbate the already challenging visualization, characterization, and quantification of infiltrative HCC. ${ }^{11,14}$ For example, regions of hyperenhancement could be due to the contrast agent uptake in the HCC, the bias field artifact, or a combination of the two.

The purpose of our study was to investigate the utility for MR bias field correction in improving response calculation among patients with infiltrative $\mathrm{HCC}$ and predict their survival after initial TACE using volumetric tumor enhancement analysis.

\section{Methods}

\section{Study cohort}

This retrospective, single-institution study (The Johns Hopkins Hospital, Baltimore, MD, USA) was compliant with the Health Insurance Portability and Accountability Act and approved by the institutional review board. Informed consent was waived. A total of 397 patients diagnosed with infiltrative $\mathrm{HCC}$ and who underwent conventional (Lipiodol ${ }^{\circledR}$ ) TACE or drug-eluting beads TACE in our institution between August 2001 and November 2013 were identified. Patients were included in this study if they underwent contrast-enhanced multi-phasic MR imaging within 1 month before and 6 weeks after TACE. Eighty-six patients were excluded because of inadequate imaging, including missing phases or imaging artifact due to motion or metal. Furthermore, 210 patients were excluded because of missing contrast agent information or use of different contrast agents between the baseline and follow-up MR. At baseline, all of the included patients were therapy-naïve with respect to systemic therapy with tyrosine kinase inhibitors and none received systemic therapy during the initial course of TACE treatment until the time point of first imaging follow-up. Finally, a total of 101 patients were included into the final analysis.

\section{TACE procedure}

The decision to treat using TACE was determined by a multidisciplinary tumor board according to our standard institutional protocol. For conventional TACE, an emulsion containing $50 \mathrm{mg}$ doxorubicin (Adriamycin; Pharmacia \& Upjohn, Peapack, NJ, USA) and $10 \mathrm{mg}$ mitomycin $\mathrm{C}$ in a 1 : $>1$ mixture with iodized oil (Lipiodol ${ }^{\circledR}$; Guerbet, Aulnay-sousBois, France) was infused and followed by $100-300-\mu \mathrm{m}$ microspheres (Embosphere Microspheres; Biosphere Medical, Rockland, MA, USA) till arterial inflow was substantially reduced. For the drug-eluting beads TACE, $100-300-\mu \mathrm{m}$ DC Beads (Biocompatible/BTG, Surrey, England) were loaded with up to $100 \mathrm{mg}$ of doxorubicin hydrochloride $(25 \mathrm{mg} / \mathrm{mL})$ and mixed with $4 \mathrm{~mL}$ of Oxilan (300 mg iodine per $\mathrm{mL}$; Guerbet, Bloomington, IN, USA) to form a $4 \mathrm{~mL}$ solution. The technical end-point was reduction of tumor arterial inflow to between 2-5 heart beats. Complete occlusion was avoided in order to maintain arterial patency for repeat treatment.

\section{MR imaging protocol}

All patients underwent MR imaging within 4 weeks before and 6 weeks after the first TACE, on a 1.5-Tesla whole-body MR scanner (Magnetom Avanto; Siemens Medical Solutions, Erlangen, Germany). A standardized MR liver protocol was used with the same imaging parameters to ensure consistency in image acquisition and timing. The imaging protocol included: (1) axial T2-weighted fast spin-echo images (TR/ TE, 5000/100 ms; matrix size, $256 \times 256$; slice thickness, 8 $\mathrm{mm}$; interslice gap, $2 \mathrm{~mm}$; receiver bandwidth, $32 \mathrm{kHz}$ ); (2) axial single-shot breath-hold gradient echo diffusionweighted echo-planar images (TR/TE, 5000-6500/110 ms; matrix size, $128 \times 128$; slice thickness, $8 \mathrm{~mm}$; interslice gap, 2 $\mathrm{mm}$; b-value, $500 \mathrm{~s} / \mathrm{mm}^{2}$; receiver bandwidth, $64 \mathrm{kHz}$ ); (3) axial breath-hold unenhanced and contrast-enhanced ( 0.1 $\mathrm{mmol} / \mathrm{kg}$ of body weight, intravenous injection of gadodiamide [Omniscan, General Electric Healthcare, Princeton, NJ, USA]) T1-weighted three-dimensional fat suppressed spoiled gradient-echo images (TR/TE, 5.1/1.2 ms; field of view, 320$400 \mathrm{~mm}^{2}$; matrix size, $192 \times 160$; slice thickness, 4-6 mm; receiver bandwidth, $64 \mathrm{kHz}$; flip angle, $15^{\circ}$ ) in the pre-contrast injection, post-contrast injection arterial, portal venous and delayed phases $(20,70$ and $180 \mathrm{~s}$ after intravenous contrast administration, respectively; a fixed time-delay technique was used). Finally, the pre-contrast and $20 \mathrm{~s}$ post-contrast scans were used for MR image processing and 3D enhancement quantification.

\section{MR image processing: Registration, segmentation and bias field correction}

All images were presented as Digital Imaging in Communications and Medicine ("DICOM") files. Image assessment was conducted independently by two radiologists (CL and SS, with 8 and 2 years of experience in abdominal MR) who did not participate in the TACE procedures and were blinded to survival outcome.

An automated 3D deformable registration (BioImage Suite, Yale University, New Haven, CT, USA) was used to register the pre- and 20 second post-contrast scans as a preprocessing step, ${ }^{15,16}$ in order to reduce inter-phase hepatic displacement and improve image quality. The whole 
liver from the arterial phase MR image was segmented in 3D semi-automatically using a prototype software which generated a 3D segmentation mask. ${ }^{8,17}$ Care was taken to exclude the major hepatic vessels, such as the hepatic artery and portal vein (Fig. 1A). Finally, the MR bias field ${ }^{18,19}$ correction using the software (BioImage Suite) to remove the intensity variation was applied to the 3D segmented liver in order to mitigate the effects of shading artifacts (Fig. 1C). This is a two-step procedure. First, the slice-by-slice inhomogeneity (shading artifacts inside and outside of the liver) in all three $(x, y, z)$ directions is normalized for the entire MR image by estimating a linear regression between any adjacent slices and removing any changes. ${ }^{19}$ Assuming that at macro-resolution $(\sim 20 \mathrm{~mm})$ the liver intensity is approximately homogeneous, the liver (as defined by the liver segmentation above) in the resultant image above is divided by a heavily blurred (macro-resolution) version of itself in order to eliminate additional intensity inhomogeneity (Fig. 1D).

\section{D enhancement quantification}

From the whole liver 3D segmentation, the ETV of the entire liver was calculated ( $q E A S L$ ) three times, and the results averaged for before and after bias field correction. This validated method has high inter-reader reproducibility and
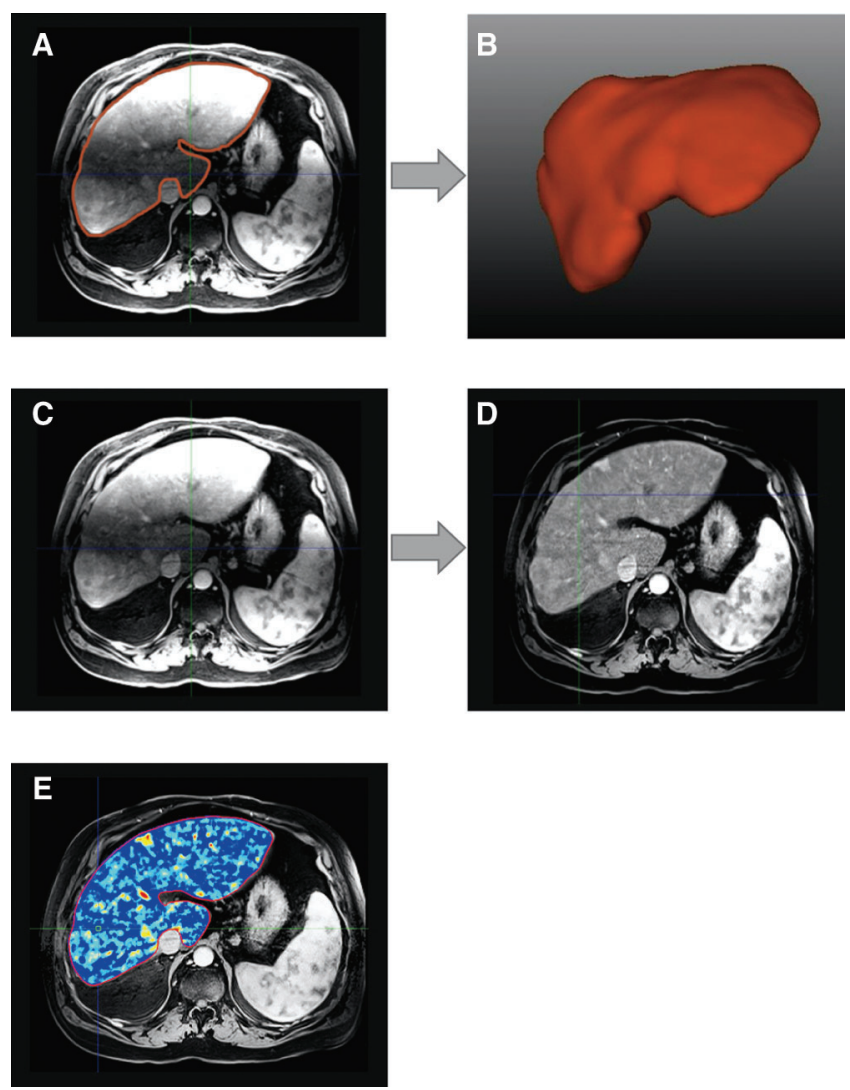

Fig. 1. Image processing workflow.

Whole liver 3D segmentation (red outline) on MR image (A) and its volumetric rendering (B). Before $(C)$ and after (D) MR bias field correction. (E) Enhancing tumor volume colormap overlay. Note the heterogeneous tumor enhancement. radiology-pathology validation. ${ }^{8,17,20}$ Briefly, the procedure was as follows:

The 3D registered pre-contrast $T 1$-weighted $M R$ image was subtracted from the contrast-enhanced arterial phase image, in order to remove contributions of background enhancement. ${ }^{21,22}$ Second, the 3D segmentation mask of the entire liver was transposed to the subtracted images. Third, a $1-\mathrm{cm}^{3} 3 \mathrm{D}$ region of interest ${ }^{23}$ was manually placed in healthy liver parenchyma that would have a coefficient of variation less than $25 \%$. Care was taken to place the region of interest away from tumors, blood vessels, gallbladder, and liver capsule during region of interest placement. Viable tumor tissue was defined as enhancement that was more than twice the standard deviation of the region of interest average signal intensity. ${ }^{23} \mathrm{~A}$ color map visualization in which the brightness and color indicating the strength and location of the enhanced signal (Fig. 1E) was also made. To improve results, we repeated the ETV calculation three times based on three different region of interest placements for each patient and the three ETV measurements were averaged. Based on the ETV results, the absolute and relative change in ETV was calculated as:

Absolute ETV change = Baseline ETV - ETV after TACE

Relative ETV change $(E T V \%)=$ Absolute ETV change / Baseline ETV

The ETV measurements/calculations were carried out with and without MR bias field correction.

\section{Statistical analysis}

The baseline characteristics were summarized using descriptive statistics. Paired $t$-test was used to compare differences with and without MR bias field correction within the same patient. OS, measured from the time of TACE to death, was estimated using the Kaplan-Meier method. Cut-off points were calculated to identify Rs and NRs. ${ }^{24}$ Differences between response groups were assessed using log-rank test. Univariate and multivariate Cox modelings were performed to identify prognostic factors. A two-sided $p$-value of less than 0.05 was considered statistically significant. All statistical analyses were carried out using SAS 9.4 (SAS Institute, Cary, NC, USA).

\section{Results}

\section{Patient characteristics}

The mean age at TACE treatment was 60.3 years for the 101 patients included in our study and 76 of them (75\%) were male. Among them, 42 patients (42\%) had portal venous invasion and 22 patients (22\%) had extra-hepatic metastasis. Also, 67 patients were treated using Lipiodol ${ }^{\circledR}$ and 34 patients using drug-eluting beads. The mean time (standard deviation) between baseline imaging and the first TACE procedure was 13.36 (13.95) days, and the mean time between TACE and follow-up imaging was 26.88 (9.23) days. None of the patients died before the first follow-up. Patients were followed up for a median (interquartile range) of 10.8 (4.0-20.7) months. The median OS of the study cohort was 12.8 months (95\% confidence interval: $10.29-16.08$ ) and by the end of observation date (February 2016), 78 patients (77\%) were deceased. Additional baseline characteristics are summarized in Table 1. 
Liu C. et al: Infiltrative HCC patient outcome prediction

Table 1. Baseline patients and tumor characteristics

\begin{tabular}{|c|c|}
\hline Variables & $n(\%)$ \\
\hline \multicolumn{2}{|l|}{ Demographics } \\
\hline Total number of patients & 101 \\
\hline \multicolumn{2}{|l|}{ Age at baseline } \\
\hline$<65$ years & $72(71)$ \\
\hline$\geq 65$ years & $29(29)$ \\
\hline \multicolumn{2}{|l|}{ Sex } \\
\hline Male & $76(75)$ \\
\hline Female & $25(25)$ \\
\hline \multicolumn{2}{|l|}{ Vital status at time of analysis } \\
\hline Alive & $23(22)$ \\
\hline Deceased & $78(78)$ \\
\hline \multicolumn{2}{|l|}{ Follow-up time in months } \\
\hline Mean (range) & $17.1(0.1-140.4)$ \\
\hline Median (interquartile range) & $10.8(4.0-20.7)$ \\
\hline \multicolumn{2}{|l|}{ Etiology } \\
\hline Hepatitis B virus & $16(16)$ \\
\hline Hepatitis C virus & $45(45)$ \\
\hline Hepatitis B virus+hepatitis C virus & $2(2)$ \\
\hline Human immunodeficiency virus & $5(5)$ \\
\hline Alcohol & $35(35)$ \\
\hline Non-alcoholic steatohepatitis & $7(7)$ \\
\hline Cryptogenic & $7(7)$ \\
\hline Cirrhosis & $36(36)$ \\
\hline \multicolumn{2}{|l|}{ Ethnicity } \\
\hline White & $65(64)$ \\
\hline African American & $16(16)$ \\
\hline Hispanic & $1(1)$ \\
\hline Other & $8(8)$ \\
\hline Unknown & $11(11)$ \\
\hline \multicolumn{2}{|l|}{ Barcelona Clinic Liver Cancer stage } \\
\hline B & $29(29)$ \\
\hline $\mathrm{C}$ & $69(68)$ \\
\hline $\mathrm{D}$ & $3(3)$ \\
\hline \multicolumn{2}{|l|}{ Child-Pugh class } \\
\hline A & $68(67)$ \\
\hline B & $32(32)$ \\
\hline $\mathrm{C}$ & $1(1)$ \\
\hline \multicolumn{2}{|l|}{ ECOG performance score } \\
\hline 0 & $56(55)$ \\
\hline 1 & 39 (39) \\
\hline 2 & $4(4)$ \\
\hline 3 & $2(2)$ \\
\hline \multicolumn{2}{|l|}{ Tumor characteristics } \\
\hline \multicolumn{2}{|l|}{ Number of lesions } \\
\hline 1 & $18(18)$ \\
\hline
\end{tabular}

Table 1. (continued)

\begin{tabular}{ll}
\hline Variables & $n(\%)$ \\
\hline 2 & $19(19)$ \\
3 & $11(11)$ \\
4 or more & $53(52)$ \\
Tumor size in cm & \\
Mean (range) & $8.6(1.5-19.5)$ \\
Median (interquartile range) & $8.0(5.0-11.5)$ \\
Portal invasion & \\
Yes & $42(42)$ \\
No & $59(58)$ \\
Extrahepatic invasion & \\
Yes & $22(22)$ \\
No & $79(78)$ \\
TACE type & \\
Lipiodol & \\
Drug-eluting beads & $67(66)$ \\
Number of TACE sessions & $34(34)$ \\
1 & \\
2 & $48(48)$ \\
3 & $19(18)$ \\
4 or more & $13(13)$ \\
\hline
\end{tabular}

\section{Imaging analysis and volumetric calculation}

Paired $t$-test was used to compare the ETV with and without MR bias field correction. As shown in Table 2, the mean baseline ETV was $823.98 \mathrm{~cm}^{3}$ without bias field correction and $502.71 \mathrm{~cm}^{3}$ with bias field correction $(p<0.001)$; similarly, after TACE treatment, mean ETV was $629.91 \mathrm{~cm}^{3}$ and $322.05 \mathrm{~cm}^{3}(p<0.001)$ with and without MR bias field correction, respectively, denoting that the application of MR bias field could impact the evaluation and the measurement of the tumor. Furthermore, we also calculated absolute ETV change and relative ETV change (ETV\%). Without MR bias field correction, mean absolute ETV decrease was 194.06 $(-1012.29-1671.84) \mathrm{cm}^{3}$ and mean ETV\% was $0.10(-5.35-$ $0.83)$, whereas with MR bias field correction, mean absolute ETV decrease was $180.66(27.08-627.70) \mathrm{cm}^{3}$ and mean ETV $\%$ was 0.37 (0.06-0.62), respectively.

With the results above, the Cox proportional hazards model was used to determine whether ETV or volume change would influence prognosis of patients with infiltrative HCC. As shown in Table 3, both baseline ETV and ETV\% with MR bias field correction correlated with prognosis. Without MR bias field correction, only baseline ETV was correlated with prognosis. Therefore, subsequent analysis was done with MR bias field corrected images only. First, the cut-off points for baseline ETV and ETV\% for prognosis prediction were identified. To determine such cut-off points for baseline ETV and ETV\% with MR bias field correction, the Q statistics test was used. The LOESS smoothed line of Martingale residuals was not straight nor close to zero, which implied that it would be better to covert ETV or ETV\% into an indicator with appropriate cut-off points instead of keeping them linear in the Cox 
Liu C. et al: Infiltrative HCC patient outcome prediction

Table 2. Comparison of ETV with and without MR bias field correction

\begin{tabular}{|c|c|c|c|}
\hline & \multicolumn{2}{|l|}{ Mean (range) in $\mathrm{cm}^{3}$} & \multirow[b]{2}{*}{$p$} \\
\hline & Without MR bias field correction & With MR bias field correction & \\
\hline Baseline ETV & $823.98(62.52-3560.86)$ & $502.71(141.41-1294.27)$ & $<0.001$ \\
\hline ETV after TACE & $629.91(46.70-3380.53)$ & $322.05(109.50-1016.39)$ & $<0.001$ \\
\hline
\end{tabular}

Table 3. Hazard ratio for baseline ETV, absolute ETV change and ETV\% with and without MR bias field correction

\begin{tabular}{|c|c|c|c|c|}
\hline \multirow[b]{2}{*}{ Variables } & \multicolumn{2}{|c|}{ Without MR bias field correction } & \multicolumn{2}{|c|}{ With MR bias field correction } \\
\hline & Hazard ratio $(95 \% \mathrm{CI})$ & $P$ & Hazard ratio $(95 \% \mathrm{CI})$ & $P$ \\
\hline Baseline ETV & $1.061(1.029,1.093)$ & $<0.001$ & $1.161(1.066,1.265)$ & $<0.001$ \\
\hline Absolute ETV change & $1.010(0.982,1.039)$ & 0.49 & $1.038(0.932,1.157)$ & 0.50 \\
\hline ETV\% & $1.003(0.979,1.028)$ & 0.79 & $0.663(0.535,0.822)$ & $<0.001$ \\
\hline
\end{tabular}

Main findings: $\bullet$ Both baseline ETV and ETV\% are factors related to prognosis for MR bias field corrected images, whereas only baseline ETV was a prognostic factor for images without MR bias field correction. $\bullet$ With MR bias field correction, when baseline ETV increased by $100 \mathrm{~cm}^{3}$, the mortality rate increased by about $16 \% . \bullet$ With MR bias field correction, when ETV\% decreased by $10 \%$, the mortality rate decreased by about $34 \%$.

model. Therefore, cut-off points were calculated with the method from Mandrekar's paper ${ }^{21}$ to identify Rs and NRs. For baseline ETV, the cut-off point was $415 \mathrm{~cm}^{3}(p=0.003)$ (Fig. $\left.2 A\right)$, and for ETV\% the cut-off point was $41 \%(p=0.01)$ (Fig. $2 \mathrm{~B})$.

\section{Survival analysis}

With the cut-off points determined, the patients were separated into different groups to check whether the cut-off points could define Rs and NRs to the treatment. As illustrated in Fig. 3A, 43 patients $(42.6 \%)$ were in the 'Baseline ETV $<415$ $\mathrm{cm}^{3 \prime}$ group and 58 patients (57.4\%) were in the 'Baseline ETV $\geq 415 \mathrm{~cm}^{3 \prime}$ group; median survival time for them was 19.66 (15.58-31.79) months and 9.21 (6.21-11.64) months, respectively $(p<0.001)$. Fifty-six patients $(55.4 \%)$ were in the 'ETV\% <41\%' group and forty-five patients (44.6\%) were in the 'ETV\% $\geq 41 \%$ ' group; median survival time for them was $8.71(6.12-11.21)$ months and 19.20 (14.5321.17 ) months, respectively ( $p=0.008)$ (Fig. 3B).

\section{Univariate and multivariate analyses}

Univariate and multivariate analyses were carried out following adjustment for factors such as age, gender, ethnicity, TACE type (Lipiodol ${ }^{\circledR}$ or drug-eluting beads), Barcelona Clinic Liver Cancer stage and Child-Pugh score. Baseline ETV and ETV\% were identified to be prognostic factors for patients with infiltrative HCC. According to the univariate analysis, the mortality rate for patients in the 'Baseline ETV $\geq 415$ $\mathrm{cm}^{3 \prime}$ group was twice as high as those in the 'Baseline ETV $\geq 415 \mathrm{~cm}^{3}$ group $(p=0.01)$ (Supplementary Table 1$)$. The mortality rate for patients in the 'ETV\% $<41 \%$ ' group was $42 \%$ lower than patients in group 'ETV\% $<41 \%$ ' $(p=0.02)$ (Supplementary Table 2 ). Of note, univariate analysis did not demonstrate the type of TACE (Lipiodol ${ }^{\circledR}$ or drug-eluting beads) to be a statistically significant prognostic factor.

\section{Discussion}

This study concludes that with MR bias field correction, it is possible to use whole liver tumor enhancement analysis to predict treatment response from baseline MR and survival after initial TACE in patients with infiltrative HCC. Specifically, baseline ETV could be used to identify candidates who may benefit from TACE before treatment, and ETV\% change after first TACE could be used to predict survival.

Characterizing infiltrative HCC is challenging because it frequently presents as a large, diffuse process. ${ }^{3-5}$ As such, image assessment of infiltrative HCC is typically done through visual observation, without objective quantitative method and standard. In a previous study, ${ }^{25}$ the image intensity of the lesion was measured in 2D and they were unable to predict survival and treatment response. In our work, the measurement is based on the whole liver in 3D and we were able to find statistical significance in predicting survival and therapy response even though we used an imaging method (i.e. MR) that has non-calibrated image intensities (as opposed to calibrated intensities like Hounsfield Units in computed tomography). The main feature is that our assessment was done on a whole liver basis using MR bias field correction. The intensity inhomogeneity observed in MR images due to the bias field produced by imperfections in the radio frequency coil, eddy currents, as well as object susceptibility make quantitative image analysis challenging. ${ }^{12-14}$ Overcoming this limitation was essential to mitigating the influence of the bias field and in doing so enabled statistically significant response and survival predictions. Our hypothesis was tested by comparing the survival and response predictive ability of volumetric enhancement with and without MR bias field correction. It was found that with bias correction, both ETV at baseline and ETV\% change after TACE could be used as predictive factors for prognosis; whereas, without MR bias field correction, only baseline ETV could predict prognosis.

In our previous work, ${ }^{8,9}$ qEASL was used for volumetric measurement of unifocal and multifocal HCC and it was determined that a volumetric ETV threshold of $65 \mathrm{~cm}^{3}$ for unifocal $\mathrm{HCC}$ and $45 \mathrm{~cm}^{3}$ for multifocal HCC could predict response. However, in the current study, the previous cut-offs could not be applied due to the characteristics of infiltrative HCC. Thus, a unique cut-point determination method using Q-statistics ${ }^{24}$ was employed to determine the cut-off for baseline ETV and ETV\% that could predict treatment response in patients with 
Liu C. et al: Infiltrative HCC patient outcome prediction
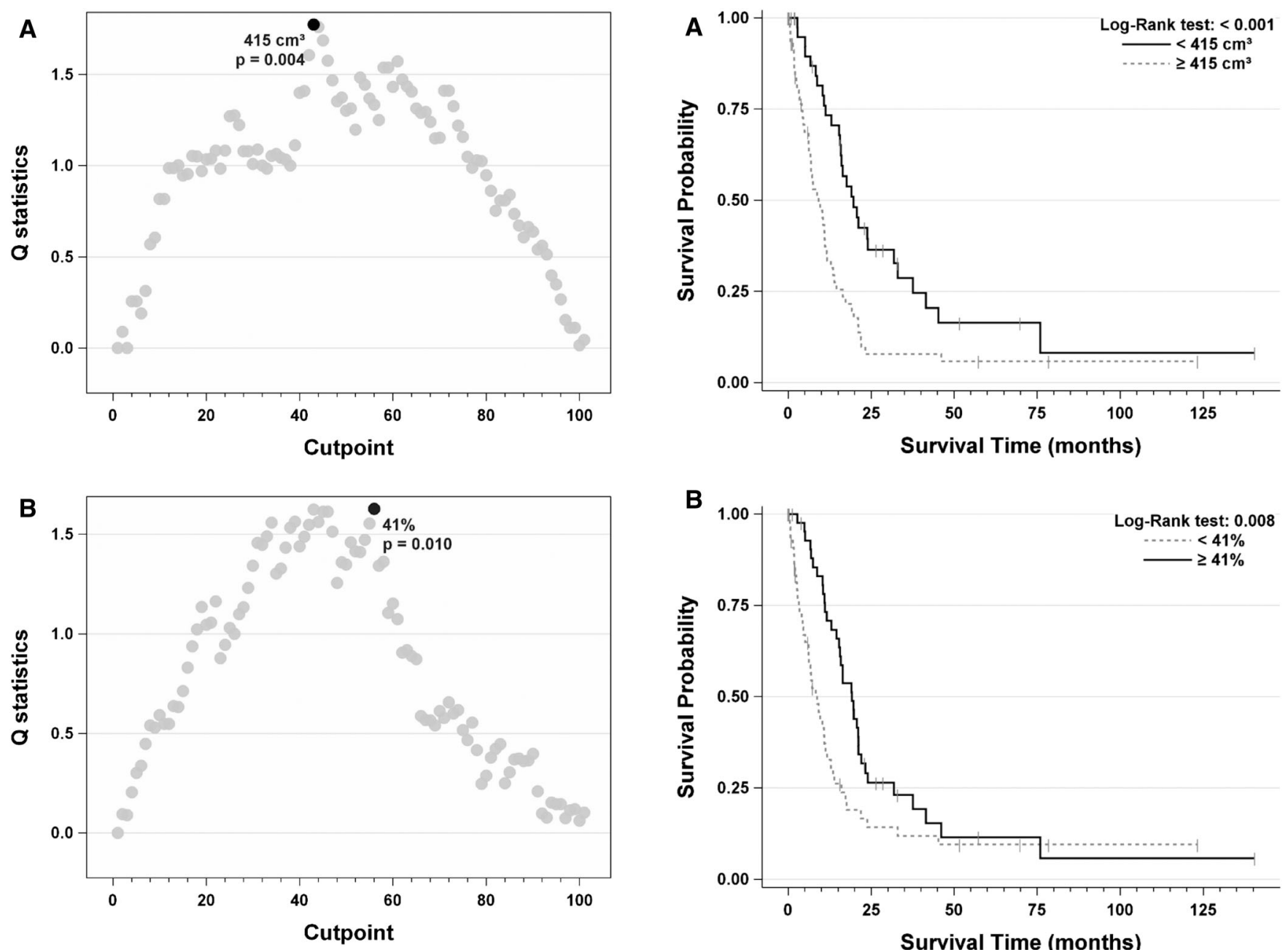

Fig. 2. Cut-off points for MR bias field corrected baseline ETV and ETV\%.

(A) ETV was $415 \mathrm{~cm}^{3}(p=0.003)$ and (B) ETV\% was $41 \%(p=0.010)$, respectively.

infiltrative HCC. It was found that patients with a baseline ETV less than $415 \mathrm{~cm}^{3}$ of infiltrative HCC tumor burden could be better candidates for TACE, and for after TACE treatment, that patients with ETV\% over $41 \%$ were found to have a longer survival time. This information can help to identify patients with infiltrative HCC who could benefit from treatments other than TACE before initiating therapy. It could further help identify NRs to TACE so that changes in therapy management could happen sooner, especially for those patients who failed to respond to the initial and second TACE. ${ }^{26}$ Additionally, these findings could help identify patients that would potentially benefit from earlier onset of systemic therapy (e. g., with sorafenib or checkpoint inhibitors) despite being formally classified as Barcelona Clinic Liver Cancer stage B.

There are several limitations to the current study. First, it was a single-institutional retrospective study with a limited cohort size. Second, the whole tumor signal is summarized into one result. Although the main branches of the portal vein and hepatic artery were excluded, smaller vessels with intensity similar to the tumor were inevitably included, possibly leading to unspecific background signal.

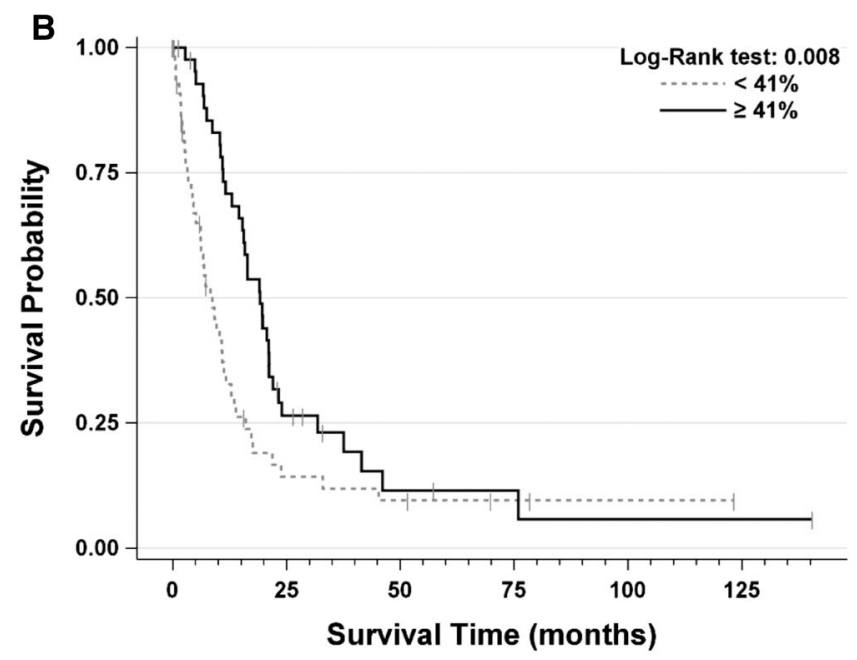

Fig. 3. Kaplan-Meier survival curves for baseline ETV and ETV\%.

The cut-offs were $415 \mathrm{~cm}^{3}$ and $41 \%$ for choosing TACE candidates and predicting treatment response, respectively.

In conclusion, MR bias field correction has the potential to improve 3D quantitative tumor assessment techniques and may add to the utility of these techniques in predicting both response and survival outcomes after initial TACE in patients with infiltrative HCC using whole liver tumor enhancement analysis. Having this predictive information, especially at time of staging/baseline imaging, could potentially improve allocation of patients to a more appropriate TACE schedule or therapy selection early during the clinical decision making process.

\section{Funding}

This research was funded by grant from NIH/NCI (R01 CA160771).

\section{Conflict of interest}

MingDe Lin is a former employee of Philips Research North America and is now an employee of Visage Imaging, Inc. Julius Chapiro declares relation to Koninklijke Philips, Guerbet SA, Eisai Co. Xenophon Papademetris declares relation to 
Brain Electrophysiology Laboratory Company (no relation to this work). The other authors have no conflict of interests related to this publication.

\section{Author contributions}

Study design ( $C L, S S, M L, J C)$, performance of experiments $(C L, S S, X P, D D M)$, analysis and interpretation of data $(C L$, $\mathrm{SS}, \mathrm{GG}, \mathrm{YD}, \mathrm{ML}, \mathrm{JC})$, manuscript writing (CL, SS, $X P, \mathrm{GG}, \mathrm{YD}$, $M L, J C, C G, K H)$, critical revision of the manuscript $(C L, M L$, $J C)$, statistical analysis (GG, YD), critical funding ( $M L, J C)$, administration (XW, CG, KH), technical or material support $(M L, X P)$.

\section{References}

[1] EASL Clinical Practice Guidelines: Management of hepatocellular carcinoma. J Hepatol 2018;69:182-236. doi: 10.1016/j.jhep.2018.03.019.

[2] Forner A, Llovet JM, Bruix J. Hepatocellular carcinoma. Lancet 2012;379: 1245-1255. doi: 10.1016/S0140-6736(11)61347-0.

[3] Sereni CP, Rodgers SK, Kirby CL, Goykhman I. Portal vein thrombus and infiltrative HCC: a pictoral review. Abdom Radiol (NY) 2017;42:159-170. doi: 10.1007/s00261-016-0855-z.

[4] Reynolds AR, Furlan A, Fetzer DT, Sasatomi E, Borhani AA, Heller MT, et al. Infiltrative hepatocellular carcinoma: what radiologists need to know. Radiographics 2015;35:371-386. doi: 10.1148/rg.352140114.

[5] Kneuertz PJ, Demirjian A, Firoozmand A, Corona-Villalobos C, Bhagat N, Herman J, et al. Diffuse infiltrative hepatocellular carcinoma: assessment of presentation, treatment, and outcomes. Ann Surg Oncol 2012;19:28972907. doi: 10.1245/s10434-012-2336-0.

[6] Demirjian A, Peng P, Geschwind JF, Cosgrove D, Schutz J, Kamel IR, et al. Infiltrating hepatocellular carcinoma: seeing the tree through the forest. J Gastrointest Surg 2011;15:2089-2097. doi: 10.1007/s11605-011-1614-7.

[7] Dai QS, Gu HL, Ye S, Zhang Y], Lin X], Lau WY, et al. Transarterial chemoembolization vs. conservative treatment for unresectable infiltrating hepatocellular carcinoma: A retrospective comparative study. Mol Clin Oncol 2014; 2:1047-1054. doi: 10.3892/mco.2014.391

[8] Fleckenstein FN, Schernthaner RE, Duran R, Sohn JH, Sahu S, Zhao Y, et al. 3D Quantitative tumour burden analysis in patients with hepatocellular carcinoma before TACE: comparing single-lesion vs. multi-lesion imaging biomarkers as predictors of patient survival. Eur Radiol 2016;26:3243-3252. doi: 10.1007/s00330-015-4168-3.

[9] Chapiro J, Duran R, Lin M, Schernthaner RE, Wang Z, Gorodetski B, et al. Identifying staging markers for hepatocellular carcinoma before transarterial chemoembolization: Comparison of three-dimensional quantitative versus non-three-dimensional imaging markers. Version 2. Radiology 2015;275: 438-447. doi: 10.1148/radiol.14141180.

[10] Tacher V, Lin M, Duran R, Yarmohammadi H, Lee H, Chapiro J, et al. Comparison of existing response criteria in patients with hepatocellular carcinoma treated with transarterial chemoembolization using a 3D quantitative approach. Version 2. Radiology 2016;278:275-284. doi: 10.1148/radiol. 2015142951.

[11] Zhao Y, Duran R, Bai W, Sahu S, Wang W, Kabus S, et al. Which criteria applied in multi-phasic CT can predict early tumor response in patients with hepatocellular carcinoma treated using conventional TACE: RECIST, mRECIST, EASL or qEASL? Cardiovasc Intervent Radiol 2018;41:433-442. doi: $10.1007 / \mathrm{s} 00270-017-1829-4$.

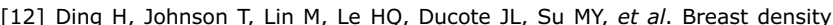
quantification using magnetic resonance imaging (MRI) with bias field correction: a postmortem study. Med Phys 2013;40:122305. doi: 10.1118/1. 4831967.
[13] Zhang $\mathrm{K}$, Liu $\mathrm{Q}$, Song $\mathrm{H}$, Li X. A variational approach to simultaneous image segmentation and bias correction. IEEE Trans Cybern 2015;45:1426-1437. doi: $10.1109 /$ TCYB.2014.2352343.

[14] Despotović I, Goossens B, Philips W. MRI segmentation of the human brain: challenges, methods, and applications. Comput Math Methods Med 2015; 2015:450341. doi: 10.1155/2015/450341.

[15] Dura E, Domingo J, Ayala G, Martí-Bonmatí L. Evaluation of the registration of temporal series of contrast-enhanced perfusion magnetic resonance 3D images of the liver. Comput Methods Programs Biomed 2012;108:932-945. doi: 10.1016/j.cmpb.2012.04.015.

[16] Chitphakdithai N, Chiang VL, Duncan JS. Non-rigid registration of longitudinal brain tumor treatment MRI. Conf Proc IEEE Eng Med Biol Soc 2011;2011: 4893-4896. doi: 10.1109/IEMBS.2011.6091212.

[17] Sahu S, Schernthaner R, Ardon R, Chapiro J, Zhao Y, Sohn JH, et al. Imaging biomarkers of tumor response in neuroendocrine liver metastases treated with transarterial chemoembolization: Can enhancing tumor burden of the whole liver help predict patient survival? Version 2. Radiology $2017 ; 283$ : 883-894. doi: 10.1148/radiol.2016160838.

[18] Zhang $Y$, Brady M, Smith S. Segmentation of brain MR images through hidden Markov random field model and the expectation-maximization algorithm. IEEE Trans Med Imaging 2001;20:45-57. doi: 10.1109/42.906424.

[19] Petersen KF, Dufour S, Savage DB, Bilz S, Solomon G, Yonemitsu S, et al. The role of skeletal muscle insulin resistance in the pathogenesis of the metabolic syndrome. Version 2. Proc Natl Acad Sci U S A 2007;104:12587-12594. doi: 10.1073/pnas.0705408104.

[20] Chapiro J, Wood LD, Lin M, Duran R, Cornish T, Lesage D, et al. Radiologicpathologic analysis of contrast-enhanced and diffusion-weighted MR imaging in patients with HCC after TACE: diagnostic accuracy of 3D quantitative image analysis. Version 2. Radiology 2014;273:746-758. doi: 10 1148 /radiol.14140033.

[21] Duran R, Chapiro J, Frangakis C, Lin M, Schlachter TR, Schernthaner RE, et al. Uveal melanoma metastatic to the liver: The role of quantitative volumetric contrast-enhanced MR imaging in the assessment of early tumor response after transarterial chemoembolization. Transl Oncol 2014;7:447 455. doi: 10.1016/j.tranon.2014.05.004.

[22] Lin M, Pellerin O, Bhagat N, Rao PP, Loffroy R, Ardon R, et al. Quantitative and volumetric European Association for the Study of the Liver and Response Evaluation Criteria in Solid Tumors measurements: feasibility of a semiautomated software method to assess tumor response after transcatheter arterial chemoembolization. J Vasc Interv Radiol 2012;23:1629-1637. doi: 10. 1016/j.jvir.2012.08.028.

[23] Chockalingam A, Duran R, Sohn JH, Schernthaner R, Chapiro J, Lee H, et al. Radiologic-pathologic analysis of quantitative $3 \mathrm{D}$ tumour enhancement on contrast-enhanced MR imaging: a study of ROI placement. Eur Radiol 2016;26:103-113. doi: 10.1007/s00330-015-3812-2.

[24] Mandrekar JN, Mandrekar SJ, Cha SS. Cutpoint determination methods in survival analysis using SAS ${ }^{\circledR}$. Statistics and Data Analysis. Available from: http://support.sas.com/resources/papers/proceedings/proceedings/sugi28/261-28.pdf.

[25] Koulakian H, Allaham W, Vilgrain V, Ronot M. Non-measurable infiltrative HCC: is post-contrast attenuation on CT a sign of tumor response? Eur Radiol 2019;29:4389-4399. doi: 10.1007/s00330-018-5805-4.

[26] Georgiades C, Geschwind JF, Harrison N, Hines-Peralta A, Liapi E, Hong K, et al. Lack of response after initial chemoembolization for hepatocellular carcinoma: does it predict failure of subsequent treatment? Version 2. Radiology 2012;265:115-123. doi: 10.1148/radiol.12112264.

[27] Treilhard J, Smolka S, Staib L, Chapiro J, Lin M, Shakirin G, et al. Liver tissue classification in patients with hepatocellular carcinoma by fusing structured and rotationally invariant context representation. Med Image Comput Comput Assist Interv 2017;10435:81-88. doi: 10.1007/978-3-31966179-7_10.

[28] Zhang F, Yang J, Nezami N, Laage-Gaupp F, Chapiro J, De Lin M, et al. Liver tissue classification using an auto-context-based deep neural network with a multi-phase training framework. Patch Based Tech Med Imaging (2018) 2018;11075:59-66. doi: 10.1007/978-3-030-00500-9_7. 